Research Square
administrative data: a
Yelena Petrosyan
Ottawa Hospital Research Institute
Kednapa Thavorn ( $\boldsymbol{D}$ kthavorn@ohri.ca )
Ottawa Hospital Research Institute
Glenys Smith
Institute for Clinical Evaluative Sciences
Malcolm Maclure
University of British Columbia
Roanne Preston
University of British Columbia
Carl van Walrevan
Ottawa Hospital Research Institute
Alan Forster
Ottawa Hospital Research Institute

Preprints are preliminary reports that have not undergone peer review. They should not be considered conclusive, used to inform clinical practice, or referenced by the media as validated information.

\title{
Predicting postoperative surgical site infection with administrative data: a Machine Learning algorithm
}

\section{Yelena Petrosyan}

Kednapa Thavorn ( $\square$ kthavorn@ohri.ca)

Ottawa Hospital Research Institute

Glenys Smith

Institute for Clinical Evaluative Sciences

Malcolm Maclure

Roanne Preston

Ottawa Hospital Research Institute

Ottawa Hospital Research Institute

\section{Research Article}

Keywords: surgical site infection, administrative data, Machine Learning, Random Forests, data mining, predictive modeling

Posted Date: December 10th, 2020

DOI: https://doi.org/10.21203/rs.3.rs-117534/v1

License: (c) (i) This work is licensed under a Creative Commons Attribution 4.0 International License.

Read Full License 


\section{Abstract}

Background: Since primary data collection can be time-consuming and expensive, surgical site infections (SSIs) could ideally be monitored using routinely collected administrative data. We derived and internally validated efficient algorithms to identify SSIs within 30 days after surgery with health administrative data, using Machine Learning algorithms.

All patients enrolled in the National Surgical Quality Improvement Program from the Ottawa Hospital were linked to administrative datasets in Ontario, Canada. Machine Learning approaches, including a Random Forests algorithm and the high-performance logistic regression, were used to derive parsimonious models to predict SSI status. Finally, a risk score methodology was used to transform the final models into the risk score system. The SSI risk models were validated in the validation datasets.

Results: Of 14,351 patients, 795 (5.5\%) had an SSI. First, separate predictive models were built for three distinct administrative datasets. The final model, including hospitalization diagnostic, physician diagnostic and procedure codes, demonstrated excellent discrimination (C statistics, $0.91,95 \% \mathrm{Cl}, 0.90$ 0.92 ) and calibration (Hosmer-Lemeshow $\chi^{2}$ statistics, 4.531, $p=0.402$ ).

Conclusion: We demonstrated that health administrative data can be effectively used to identify SSIs. Machine learning algorithms have shown a high degree of accuracy in predicting postoperative SSIs and can integrate and utilize a large amount of administrative data. External validation of this model is required before it can be routinely used to identify SSIs.

\section{Background}

Surgical site infection (SSI) is common and considered one of the most common types of postoperative complications (1). SSIs are associated with substantial morbidity and mortality, prolonged hospital duration of stay, increased hospital readmission rate, and financial burden to health care systems (1-5). Previous research has shown the importance of effective prevention strategies targeting both short- and long-term consequences of SSI, which requires an ability to track SSIs (2). Since the primary data collection can be time-consuming and expensive, routinely collected health administrative data offer ample opportunities to identify and monitor SSIs, and assess the impact of prevention strategies, given a wide population coverage and minimal costs and efforts. Several studies have developed some accurate administrative algorithms to identify SSIs (6-10), while other studies have found that SSI identification using administrative data is imprecise (11). However, previous studies were often based on small sample sizes and/or a limited set of pre-selected variables to predict SSIs.

Machine learning approaches have been successfully applied to create predictive models in several fields of study, including automatic medical diagnostics $(12,13)$. With interpretability of model parameters and ease of use, logistic regression can generate excellent models and serve as a commonly accepted statistical tool. Random Forests approach is used in situations where regression assumptions may be violated by situations in which many predictors are associated with a small number of outcomes (16). It 
can cope with inter-correlation between multiple explanatory variables, since each predictor is selected randomly for each stage of the learning process (17), unlike standard regression approaches. Previous studies have indicated that the Random Forests approach may have better prediction accuracy than other machine learning methods $(14,15)$. We hypothesized that the use of machine learning approaches and a large data set with many features will improve the accuracy of SSI prediction. This study aimed to develop efficient algorithms to identify SSIs within 30 days after surgery using health administrative data.

\section{Material And Methods}

This study was divided into three stages. In the first stage, a Random Forests algorithm was used to perform a preliminary screening of variables and to rank the importance of candidate variables. In the second stage, the 30 most important variables from the first stage were input into the high-performance logistic regression to build interpretable and parsimonious models for all three administrative datasets used in this study. Finally, we used risk score modeling methodology to transform the final logistic models form the second stage into the risk score system.

\section{Selection and Description of Participants}

This study was performed at The Ottawa hospital (TOH), Canada, a 1200-bed academic health sciences center providing approximately $90 \%$ of the major surgical operations in a catchment area of 1.2 million people. We identified all patients at $\mathrm{TOH}$ aged 18 years and older who underwent surgery and were included in the American College of Surgeons National Surgical Quality Improvement Program (NSQIP) data collection, between April 1, 2010, and March 31, 2015. The NSQIP uses trained Surgical Clinical Reviewers to collect data using a combination of chart review and follow up from the preoperative period through 30 days postoperatively. Patients were excluded if: 1) they were not eligible for the Ontario Health Insurance Program (OHIP) or had an invalid OHIP number, because this was required for linkage to health administrative datasets; or 2) they had missing admission, discharge, or surgery dates.

\section{Population-based Health Administrative Datasets}

We linked the NSQIP dataset to three distinct population-based, health administrative datasets housed at the Institute for Clinical and Evaluative Sciences (ICES). ICES is an independent, non-profit research institute whose legal status under Ontario's health information privacy law allows it to collect and analyze health care and demographic data, without informed consent, for health system evaluation and improvement. The use of data in this project was authorized under Sect. 45 of Ontario's Personal Health Information Protection Act, which does not require review by a Research Ethics Board. The datasets included: 1) the Discharged Abstract Database and Same Day Surgery Database to identify the records of the hospitalization (ICD-10 code), including admission and discharge dates, diagnoses, 2) the Physician Services Database to retrieve all claims for services provided by all eligible health care providers, and 3 ) the Ontario Health Insurance Plan (OHIP) database that contains physician diagnostic codes (ICD-9 codes) and diagnosis descriptions. All patients were followed for 30 days from the time of their surgery. All databases were linked using anonymized unique identifiers and analyzed at the ICES at the University 
of Ottawa, Ontario. This study was approved by the Ottawa Health Science Network Research Ethics Board.

\section{Study outcome}

All individuals who had any type of SSIs (i.e. superficial, deep, or organ space) (Additional file 1) within 30 days after surgery, according to the definition of the NSQIP protocol, were defined as having experienced an SSI.

\section{Statistical analysis}

This study utilized a 3-stage predictive modeling based on the hybrid modeling approaches developed in previous studies (16-18). All stages described below were applied to each administrative dataset used in this study to generate three sub-models that contributed to the omnibus SSI model.

\section{Stage 1 - Model development using Random Forests algorithm}

Details of Random Forests method have been described elsewhere (19-21). In short, each of the classification trees is built using a bootstrap sample of the data, and a random subset of variables was selected at each split, thereby constructing a large collection of decision trees with controlled variation $(22,23)$ (Additional file 2). The Random Forests trees are not pruned, so as to obtain low-bias trees. Every tree in the forest casts a "vote" for the best classification for a given observation, and the class receiving most votes results in the prediction for that observation. The study cohort was first divided randomly into derivation (70\%) and validation (30\%) samples (Additional file 3). Then, the derivation data was sampled to create an in-bag partition - (2/3) to construct the decision tree, and a smaller out-of-bag partition (1/3) to test the constructed tree to evaluate its performance by computing: 1) misclassification error, 2) Cstatistics, and 3) model performance (sensitivity, specificity, etc.). The optimal number of trees and a subset of variables at each node were selected using the "tuneRF" function in R to minimize the misclassification error. Random Forests calculates estimates of variable importance for classification using permutation variable importance measure (VIM) (19), which is based on the decrease of a classification accuracy when values of a variable in a node of a tree are permuted randomly. Finally, Kfold cross validation was used to evaluate the Random Forests model with 10 folds. We identified subsets of top 30 important diagnostic or procedure codes to predict SSIs, using a mean decrease accuracy value of 0.02 as a cut-off point. The Random Forests analyses were performed in R statistical software (3.3.2.) using "randomForest" package (21).

\section{Stage 2 - Stepwise model selection using high-performance logistic regression approach}

The 30 most variables identified after Random Forests were input into the high-performance logistic model with stepwise variable selection to find the best parsimonious model to predict SSIs (24). The Schwarz Bayesian Criterion (SBC) was used as a penalized measure of fit for logistic regression model to help avoid the model over-fitting.

\section{Stage 3 - Risk score modeling approach}

We used the methods suggested by Sullivan et al (25) to summarize each logistic model from stage 2 as a point system. The point scores were developed for hospitalization (ICD-10) and physician (ICD-9) 
diagnostic codes, and physician procedure claims. All variables in the models were categorical, and the distance between a variable and its base category in regression coefficient units was equal to the size of the coefficient. For each variable, its distance from the base category in regression coefficient units was divided by this constant and rounded to the nearest integer to get its point value.

Then, the obtained point scores were input into logistic regression model and adjusted for other potential confounding factors suggested by the existing literature, including age, sex, surgical procedure, emergency case, concurrent surgical procedures, patient's physical status (ASA-5), and duration of surgery. The full model discrimination (C statistics or AUC) and calibration (Hosmer-Lemeshow (H-L) statistics) were assessed in the validation dataset. All methods were performed in accordance with the guidelines for developing and reporting Machine Learning predictive models in biomedical research (26). The high-performance regression and point score assignment were performed in SAS 9.4 statistical software.

\section{Results}

We identified 14,351 patients who underwent surgery from April 1, 2010 to March 31, 2015 and were enrolled into NSQIP at our hospital. An SSI was identified in $795(5.5 \%)$ of these patients. Of these, 540 (68\%) had superficial SSIs and 255 (32\%) had deep or organ space SSIs. Descriptive statistics for patients in the study sample are reported in Additional file 4. The derivation and validation datasets were similar in terms of baseline covariates (Additional file 5).

\section{Predictive modeling for hospitalization diagnostic codes (ICD-10)}

We identified 3,085 hospitalization diagnostic (ICD-10) codes recorded within 30 days following the surgery date. These codes then were clustered into 994 three-digit hospitalization diagnostic codes that were used for the further analyses.

Stage 1: Given a large number of diagnostic codes (possible predictors), the Random forests approach was used to identify a subset of top important 30 hospitalization diagnostic codes that best predicts classification. We used 800 classification trees and 46 variables available for splitting at each tree node. The accuracy of the Random Forests model was $95.3 \%$. The resulting SSI prediction model demonstrated positive predictive value (PPV) of $98 \%$, negative predictive value (NPV) of $97 \%$, and AUC (area under the receiver operating characteristic curve) of 0.78 (95\% $\mathrm{Cl} 0.77-0.79)$. The accuracy of the Random Forests model after a 10 -fold cross-validation was $94.3 \%$. Figure 1 presents the top 30 hospitalization diagnostic (ICD-10) codes for classification of SSIs that have been identified using the permutation VIM.

T81 - Operative complication (infection, hemorrhage, etc.); C54 - Malignant neoplasm of specified part of uterus; K65 - Peritonitis; B96 - Other bacterial agents as the cause of diseases classified elsewhere; K83 - Biliary duct infection, obstruction, perforation, or fistulation; Y83 - Surgical operation/procedures as the cause of abnormal reaction of the patient/or later complication; C51 - Malignant neoplasms of female genital organs; Y83 - Surgical operation/procedures as the cause of abnormal reaction of the 
patient/complication; C51 - Malignant neoplasms of female genital organs; K75 - Abscess of liver; L27 Dermatitis and eczema; B95 - Streptococcus and staphylococcus as the cause of diseases; K42 Umbilical hernia; A04 - Other bacterial intestinal infections; M71 - Bursal abscess, cyst, infection; N39 Other disorders of urinary system; D05 - Carcinoma in situ of breast; C21 - Malignant neoplasm of anus and anal canal; T85 - Complications of internal prosthetic devices, implants and grafts; K26 - Duodenal ulcer; N43 - Other disorders of prostate; C25 - Malignant neoplasm of pancreas; A49 - Bacterial infection of unspecified site; K35 - Acute appendicitis; K92 - Other diseases of digestive system; K63 - Other diseases of intestine; K55 - Vascular disorders of intestine; G00 - Bacterial meningitis, unspecified; Y60 Unintentional cut, puncture, perforation or haemorrhage during surgical and medical care; D62 - Acute posthaemorrhagic anemia; J80 - Acute respiratory distress syndrome

Stage 2: The identified top 30 hospitalization diagnostic codes (ICD-10) codes were input into the highperformance logistic regression with a stepwise selection to identify the best parsimonious model to predict SSIs. Table 1, model 1 presents the final model of six hospitalization diagnostic codes to identify SSIs (AUC 0.87, 95\% Cl 0.86-0.89). 
Table 1

The best parsimonious models for prediction of SSIs

Model 1. The best parsimonious model of hospitalization diagnostic (ICD 10) codes

Effect

$\begin{array}{ll}{ }^{*} \mathrm{AOR}, 95 \% & \text { Risk } \\ \mathrm{Cl} & \text { point }\end{array}$

T81- Operative complication (infection, hemorrhage, etc.)

6.40

2

$(5.08-$

8.01)

K65 - Peritonitis

5.87

$(3.88-$

7.88)

B96 - Other bacterial agents causing infections

2.56

$(1.84-$

1

3.47)

K83 - Biliary duct infection, obstruction, perforation

6.32

$(4.42-$

8.01)

Y83 - Surgical operation/procedures as the cause of abnormal reaction of the patient/ or later complication

2.46

(1.97-

3.07)

B95 - Streptococcus/ staphylococcus as the cause of diseases

3.25

(2.17-

4.87)

Model 2. The best parsimonious model of physician diagnostic (ICD 9) codes

Effect

AOR, 95\%

Risk

$\mathrm{Cl}$

686 - Pyoderma, pyogenic granuloma, other local infections

8.13

$(6.50-$

3

$9.20)$

682-Cellulitis, abscess

4.70

(3.57-

2

6.10)

998 - Other complications of procedures

5.68

(4.77-

2

$6.78)$

556 - Ulcerative colitis

8.60

3

$(6.31-$

9.18)

685 - Pilonidal cyst with fistula, abscess

2.69

2

$(1.52-$

3.76)

*AOR, 95\% Cl = Adjusted Odds Ratio, 95\% Confidence Interval 
Model 1. The best parsimonious model of hospitalization diagnostic (ICD 10) codes

560 - Intestinal obstruction without mention of hernia

2.97

(2.19-

2

4.01)

154 - Malignant neoplasm of rectum, rectosigmoid junction

4.37

(3.29-

5.17)

$\mathbf{5 9 9}$ - Other disorders of urethra and urinary tract

2.04

$(1.55-$

1

2.62)

153 - Malignant neoplasm of colon

2.71

(2.02-

3.22)

Model 3. The best parsimonious model of physician procedure claims

Effect

AOR, 95\%

Risk

$\mathrm{Cl}$ point

Z59 - Digestive system surgical procedure: colon/biliary tract

7.38

$(6.08-$

4

9.09)

C46 - Infectious disease: hospital consult/assessment

5.77

$(4.66-$

7.43)

Z10 - Skin/subcutaneous tissue: incision of abscess or hematoma

7.88

$(6.04-$

3

8.67)

C03 - General surgery: hospital consult/assessment

3.45

$(2.86-$

4.19)

H15 - Family practice: assessment on weekend

2.33

$(1.80-$

3.01)

S16 - Digestive system surgical procedures: intestine

1.98

$(1.48-$

2.52)

C20 - Obstetrics and gynecology assessment/consult

2.25

$(1.66-$

3

$\begin{array}{ll}2.71 & 1 \\ (2.02- & \\ 3.22) & \end{array}$




\section{Model 1. The best parsimonious model of hospitalization diagnostic (ICD 10) codes}

S21 - Digestive system surgical procedures: colon/rectum

R06 - Skin/subcutaneous tissue: free island flaps

C13 - Internal medicine: hospital assessment/consult

H13 - Family practice: assessment/consult on weekdays

C21 - Pain management: limited consultations$$
\text { . }
$$

R11-Operations of the breast: incision, excision, repair
2.65

$(1.91-$

3.62)

4.64

$(2.58-$

6.36)

1.96

(1.52-

2.36)

2.85

(2.18-

3.52)

1.84

(1.55-

2.10)

2.81

(1.02-

3.41)
2

3

1

.

2

1

3

2

.

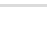


nervous system; 257 - Testicular dysfunction; 603 - Hydrocele; 560 - Intestinal obstruction without mention of hernia; 608 - Other disorders of male genital organs; 170 - Malignant neoplasm of bone and articular cartilage; 154 - Malignant neoplasm of rectum, rectosigmoid junction and anus; 821 - Fracture of femur; 075- Infectious mononucleosis, glandular fever; 917- Superficial injury of foot and toe(s); 788 Symptoms involving urinary system; 153 - Malignant neoplasm of large intestine - excluding rectum; 372 - Conjunctiva disorders (e.g., conjunctivitis, pterygium); 845 - Sprains and strains of ankle and foot; 591 - Hydronephrosis; 184 - Malignant neoplasm of vagina, vulva, other female genital organs; 156 Malignant neoplasm of gallbladder and extra hepatic bile ducts; 290 - Senile dementia, presenile dementia; 569- Other disorders of intestine; 646 - Other complications of pregnancy (e.g., vulvitis, vaginitis, cervicitis, pyelitis, cystitis); 437- Other and ill-defined cerebrovascular disease; 346 - Other diseases of central nervous system (e.g., brain abscess, narcolepsy, motor neuron disease, syringomyelia)

Stage 2: The identified top 30 physician diagnostic codes were input into the high-performance logistic regression model to identify the best parsimonious model for prediction of SSIs, using a stepwise selection approach. Table 1, Model 2 presents the final models of nine physician diagnostic codes to identify SSIs (AUC 0.85, 95\% CI 0.84-0.86).

Stage 3: Risk scores for the final model of physician diagnostic codes are presented in Table 1, Model 2 (25). Among the entire cohort, $77.8 \%$ of patients had a score of $0,7.7 \%$ had a score of 1 , and $14.5 \%$ had a score equal or greater than 2 .

\section{Predictive modeling for physician procedure claims}

We identified 2,543 physician procedure claims recorded within 30 days following the surgery date. These codes then were clustered into 610 three-digit codes that were used for the further analyses.

Stage 1: Given a large number of physician procedure codes (possible predictors), Random forests approach was used to identify a subset of 30 physician procedure claims that best predicts SSIs. The best misclassification rate was achieved by using 1,000 classification trees and 37 variables available for splitting at each tree node. The accuracy of the Random Forests model was $94.8 \%$. The resulted SSI prediction model demonstrated PPV of $99 \%$, NPV of $97 \%$, and AUC of 0.82 ( $95 \% \mathrm{Cl} 0.81-0.83$ ). The accuracy of the model after a 10 -fold cross-validation was $94.4 \%$. Figure 3 presents the top 30 physician procedure claims that have been identified using the permutation VIM.

Z59 - Digestive system surgical procedure; C46 - Infectious disease - non-emergency hospital in-patient services: assessment/ consultation; Z10 - Integumentary system surgical procedures: incision of abscess/ haematoma; K07 - Family practice/geriatrics acute and chronic home care supervision; K99 Emergency department - special visit premium; C03 - General surgery, non- emergency hospital in-patient services-assessment, visits, consultations; A35 - Urology -consultations/ assessment; S16 - Digestive system surgical procedures; $\mathrm{H} 15$ - Family practice \& practice in general - weekend and holidays: assessment/care; C64 - General thoracic surgery - non-emergency hospital in-patient services: consultation assessment; $\mathrm{H} 12$ - Family practice \& practice in general - nights assessment and car; C12Non-emergency hospital in-patient services: Subsequent visits by the MRP; R11- Integumentary system 
surgical procedures: operations of the breast; E08 - Hospital and institutional consultations/assessments by MRP; C20 - Obstetrics and gynecology - non-emergency hospital in-patient services; Z08 - Debridement of wound(s) and/or ulcer(s) extending into subcutaneous tissue, tendon, ligament, bursa and/or bone; G55- Diagnostic and therapeutic procedures, critical care; S21- Digestive system surgical procedures: rectum; S65 - Male genital surgical procedures; Z74 - Respiratory surgical procedures; R62Musculoskeletal system surgical procedures - amputation; A20 - Obstetrics and gynecology assessment or consultation; Z22 - Musculoskeletal system surgical procedures; R06 - Myocutaneous, myogenous or fascia-cutaneous flaps, neurovascular island transfer, transplantation of free island skin and subcutaneous flap; A24 - Otolaryngology - assessment/ consultation; C13 - Internal and occupational medicine: non- emergency hospital in-patient services; C01 - Non-emergency hospital inpatient services, subsequent visits by the MRP; H13 - Family practice \& practice in general -weekdays, evenings: assessment/care; C21 - Consultations/visits anaesthesia -non-emergency hospital in-patient services

Stage 2: The identified top 30 physician procedure claims were input into the high-performance logistic regression model to identify the best parsimonious model for prediction of SSIs. We used a stepwise variable selection approach. Table 1, Model 3 presents the final models of 14 physician procedure claims to identify SSIs (AUC $0.84,95 \% \mathrm{Cl} 0.83-0.85$ ).

Stage 3: Risk scores for the final model of physician procedure claims are presented in Table 1, Model 3 (25). Among the entire cohort, $55.4 \%$ of patients had a score of $0,11.9 \%$ had a score of 1 , and $44.6 \%$ had a score equal or greater than 2 .

\section{Full model with total risk score of diagnostic and procedure codes}

In the derivation cohort, the total scores of hospitalization diagnostic (ICD-10) codes, physician diagnostic (ICD-9) codes and physician procedure claims were included in the logistic regression model and adjusted for potential confounding factors, including surgical specialties, age, sex, duration of surgery, emergency case, ASA class and concurrent surgical procedures (Table 2). 
Table 2

Full model of total risk scores for hospitalization diagnostic (ICD-10) codes, physician diagnostic (ICD-9) codes and physician procedure claims, adjusted for the study

covariates

\begin{tabular}{|c|c|c|}
\hline Effect & Adjusted Odds Ratio & 95\% Confidence interval \\
\hline Hospitalization diagnostic score & 2.12 & $1.91-2.20$ \\
\hline Physician diagnostic score & 1.88 & $1.75-2.02$ \\
\hline Physician procedure score & 1.45 & $1.31-1.56$ \\
\hline Age $<65$ years & 1.74 & $1.40-2.16$ \\
\hline Log-operation duration, min & 1.52 & $1.30-1.72$ \\
\hline \multicolumn{3}{|l|}{ Surgical specialty } \\
\hline General surgery & 1.60 & $1.20-2.15$ \\
\hline Gynecology & 1.19 & $0.80-1.76$ \\
\hline Orthopedics & 0.77 & $0.53-1.11$ \\
\hline Plastics & 2.37 & $1.59-3.51$ \\
\hline Vascular & 1.75 & $1.12-2.68$ \\
\hline Other & Reference & Reference \\
\hline Female & 1.18 & $0.96-1.47$ \\
\hline \multicolumn{3}{|l|}{ Concurrent surgical procedures } \\
\hline 1 & 1.05 & $0.67-1.63$ \\
\hline $2+$ & 1.09 & $0.67-1.75$ \\
\hline 0 & Reference & Reference \\
\hline \multicolumn{3}{|l|}{ ASA class } \\
\hline I & 0.87 & $0.75-1.33$ \\
\hline II & 1.21 & $0.80-1.80$ \\
\hline III & 1.10 & $0.66-1.76$ \\
\hline IV & 0.32 & $0.04-1.03$ \\
\hline V & Reference & Reference \\
\hline Emergent case & 0.99 & $0.79-1.20$ \\
\hline
\end{tabular}


The full model had excellent discrimination (AUC 0.91; 95\% Cl, 0.90-0.92) and calibration (H-L statistics, 4.53, $p=0.402$ ). The predicted probability threshold with the optimal operating characteristics (27) (e.g., the square of distance between the point $(0,1)$ on the upper left hand corner of ROC space and any point on ROC curve) was a predicted risk of $4 \%$ (sensitivity, $83.4 \%$; specificity, $89.2 \%$; PPV, $34.2 \%$; and NPV, 99.1\%). In the internal validation cohort, the full model remained strongly discriminative (AUC $0.89,95 \%$ $\mathrm{Cl} 0.88-0.90)$ and well calibrated (H-L statistics, 6.47, $\mathrm{p}=0.487)$ (Fig. 4).

\section{Discussion}

We used a 3-stage predictive modeling approach to derive and internally validate models to predict SSIS within 30 days after surgical procedure. To the best of our knowledge, this is the first study that used Machine Learning approaches to develop efficient algorithms for identifying SSIs within 30 days after surgery by use of health administrative data. The key finding of our study is that the risk of SSIs can be reliably estimated using routinely collected administrative data, including physician procedure claims, hospital (ICD-10) and physician (ICD-9) diagnostic codes. Our study results demonstrate high performance of the Random Forests algorithm for prediction of SSIs without pre-selection of possible predictors given a small number of cases. We derived a relatively small set of variables to identify postoperative SSIs, including 6 hospital diagnostic codes, 9 physician diagnostic codes, and 14 physician procedure claims.

Several studies have examined the use of administrative data to identify postoperative SSIs (6-10). Our study findings are consistent with these studies $(6,10)$. van Walreven et al. $(6)$, for example, found that administrative data, including hospital diagnostic, emergency department visit codes and physician procedure claims, can be effectively used to identify postoperative patients with a low risk of having SSIs within 30 days of their surgical procedure. In particular, the predictive probability threshold with the optimal characteristics was a predicted risk of $5 \%$ (sensitivity, $82.1 \%$, specificity, $85.6 \%$, PPV, $27.7 \%$ ). Additionally, Sands et al. found that (9) automated medical and claim records together can be used to screen for post discharge SSIs, but the method they used identified only $10 \%$ of procedures as possible infections.

The approach used in our study added a new contribution to the existing literature by incorporating much larger set of features as compared with the previous studies. It was possible to include all available diagnostic or procedure codes to identify SSIs in this study, because Random Forests approach is generally unaffected by the addition of irrelevant features and is robust to collinearity due to the use of subsets of random variables for tree splits. All the features included in this study were obtained from routinely collected data, and given the complex etiology of SSIs, there might be variables that would be overlooked if we used a narrower search strategy guided by a priori clinical expectations. It would be inappropriate to interpret the identified diagnostic or procedure codes as either causes or consequences of SSIs. Random Forests allows us to select variables that are influencing prediction given a small sample sizes and the extremely small ratio of samples to variable (large " $p$ " and small " $n$ "). If the 
identified important variables are consistent with clinical knowledge, there will be more confidence in the derived model as a decision support tool.

Several aspects of our study should be carefully considered. First, our study contained no information about outpatient antibiotic treatments because the Ontario health administrative data used for the study captures medication use for people over the age of 65 and who are on social assistance. Also, we did not include information about laboratory tests, because the Ontario health administrative data captures information only on outpatient laboratory tests, while laboratory tests performed during hospitalization are most important in predicting SSIs. Thus, information about antibiotic use and laboratory test could substantially improve SSI identification. Second, our study and model captured SSIs that occurred within 30 days after surgical procedure, so any SSI that occurred outside of this timeframe would have been missed. Third, our study was conducted in a single teaching hospital, providing about $90 \%$ of the major surgical operations in a catchment area of 1.2 million people. Therefore, external validation is necessary to measure model's utility in other hospitals and geographic regions. Finally, the coding systems used in the province of Ontario might not be available in other jurisdictions. Therefore, some modifications might be required before using our models in other health regions.

\section{Conclusion}

This study shows that health administrative data could be effectively used in identifying SSIs. Machine learning approaches have shown a high degree of accuracy in predicting postoperative SSIs and can integrate and utilize a large amount of administrative data. The results of our study are useful in advancing current and future efforts to use administrative data for patient safety surveillance and improvement. Further research should examine the use of machine learning approaches to identify SSIs, stratified by the specific types of surgical procedures.

\section{Abbreviations}

SSI - surgical site infections; NSQIP - National Surgical Quality Improvement Program, OHIP- Ontario Health Insurance Plan; ICD - Classification of Diseases; ASA - patient's physical status; HosmerLemeshow $(\mathrm{H}-\mathrm{L})$ statistics; PPV - positive predictive value; NPV - negative predictive value; VIM - variable importance measure; AOR - adjusted odds ratio; $\mathrm{Cl}$ - confidence interval; AUC - Area under curve.

\section{Declarations}

\section{Acknowledgments}

This study was supported by ICES, which is funded by an annual grant from the Ontario Ministry of Health and Long-Term Care (MOHLTC). The opinions, results and conclusions reported in this paper are those of the authors and are independent from the funding sources. No endorsement by ICES or the Ontario MOHLTC is intended or should be inferred. 


\section{Funding}

This study was supported by the Ontario Research Fund (RE05-070) and Canadian Institutes of Health Research (ClHR) grant. The study design, opinions, results and conclusions reported in this paper are those of the authors and are independent from the funding sources.

\section{Ethics declarations}

\section{Ethics approval}

This study was approved by the Ottawa Health Science Network Research Ethics Board.

Consent to participate

Not applicable.

\section{Consent for publication}

ICES is a prescribed entity under section 45 of Ontario's Personal Health Information Protection Act. Section 45 authorizes ICES to collect personal health information, without informed consent, for the purpose of analysis or compiling statistical information with respect to the management, evaluation or monitoring of the allocation of resources to or planning for all or part of the health system. Projects conducted under section 45, by definition, do not require review by a Research Ethics Board. This project was conducted under section 45 and approved by ICES' Privacy and Legal Office.

\section{Availability of data and materials}

The data that support the findings of this study are available at the Institute for Clinical Evaluative Sciences (ICES) (www.ices.on.ca/DAS), but restrictions apply for the availability of these data, which were used under license for the current study, and so are not publicly available. Data are however available from the authors upon reasonable request and with permission of ICES.

\section{Competing interests}

No researcher involved in this study had any declared or otherwise known conflicts of interest.

\section{Author information}

\section{Authors' contributions}

Yelena Petrosyan: conception and design, analysis and interpretation of data; drafting the article and revising it critically for important intellectual content: final approval of the version to be published.

Kednapa Thavorn: conception and design, interpretation of data; critical revision of the article critically for important intellectual content; final approval of the version to be published. 
Glenys Smith: acquisition of data.

Malcolm Maclure: conception, critical revision of the article critically for important intellectual content; final approval of the version to be published.

Roanne Preston: conception and design, critical revision of the article critically for important intellectual content; final approval of the version to be published.

Carl van Walrevan: conception and design, critical revision of the article critically for important intellectual content; final approval of the version to be published.

Alan Forster: conception and design, acquisition of data, critical revision of the article critically for important intellectual content; final approval of the version to be published.

\section{References}

1. Pittet $D$, Harbarth S, Ruef $C$, Francioli $P$, Sudre $P$, Petignat $C$, et al. Prevalence and risk factors for nosocomial infections in four university hospitals in Switzerland. Infect Control Hosp Epidemiol. 1999;20(1):37-42.

2. Petrosyan Y, Thavorn K, Maclure M, Smith G, Mclsaac DI, Schramm D, et al. Long-term Health Outcomes and Health System Costs Associated With Surgical Site Infections: A Retrospective Cohort Study. Ann Surg. 2019.

3. Jenks PJ, Laurent M, McQuarry S, Watkins R. Clinical and economic burden of surgical site infection (SSI) and predicted financial consequences of elimination of SSI from an English hospital. J Hosp Infect. 2014;86(1):24-33.

4. Whitehouse JD, Friedman ND, Kirkland KB, Richardson WJ, Sexton DJ. The impact of surgical-site infections following orthopedic surgery at a community hospital and a university hospital: adverse quality of life, excess length of stay, and extra cost. Infect Control Hosp Epidemiol. 2002;23(4):183-9.

5. Badia JM, Casey AL, Petrosillo N, Hudson PM, Mitchell SA, Crosby C. Impact of surgical site infection on healthcare costs and patient outcomes: a systematic review in six European countries. J Hosp Infect. 2017;96(1):1-15.

6. van Walraven C, Jackson TD, Daneman N. Derivation and Validation of the Surgical Site Infections Risk Model Using Health Administrative Data. Infect Control Hosp Epidemiol. 2016;37(4):455-65.

7. Grammatico-Guillon L, Baron S, Gaborit C, Rusch E, Astagneau P. Quality assessment of hospital discharge database for routine surveillance of hip and knee arthroplasty-related infections. Infect Control Hosp Epidemiol. 2014;35(6):646-51.

8. Rennert-May E, Manns B, Smith S, Puloski S, Henderson E, Au F, et al. Validity of administrative data in identifying complex surgical site infections from a population-based cohort after primary hip and knee arthroplasty in Alberta, Canada. Am J Infect Control. 2018;46(10):1123-6. 
9. Sands K, Vineyard G, Livingston J, Christiansen C, Platt R. Efficient identification of postdischarge surgical site infections: use of automated pharmacy dispensing information, administrative data, and medical record information. J Infect Dis. 1999;179(2):434-41.

10. van Walraven C, Jackson TD, Daneman N. Administrative data measured surgical site infection probability within 30 days of surgery in elderly patients. J Clin Epidemiol. 2016;77:112-7.

11. Song $X$, Cosgrove S, Pass M, Perl T. Using hospital claim data to monitor surgical site infections for inpatient procedures. American Journal of Infection Control. 2008;36(3).

12. Cohen AM, Ambert K, McDonagh M. A Prospective Evaluation of an Automated Classification System to Support Evidence-based Medicine and Systematic Review. AMIA Annu Symp Proc. 2010;2010:121-5.

13. Szlosek DA, Ferrett J. Using Machine Learning and Natural Language Processing Algorithms to Automate the Evaluation of Clinical Decision Support in Electronic Medical Record Systems. EGEMS (Wash DC). 2016;4(3):1222.

14. Maroco J, Silva D, Rodrigues A, Guerreiro M, Santana I, de Mendonca A. Data mining methods in the prediction of Dementia: A real-data comparison of the accuracy, sensitivity and specificity of linear discriminant analysis, logistic regression, neural networks, support vector machines, classification trees and random forests. BMC Res Notes. 2011;4:299.

15. Douglas PK, Harris S, Yuille A, Cohen MS. Performance comparison of machine learning algorithms and number of independent components used in fMRI decoding of belief vs. disbelief. Neuroimage. 2011;56(2):544-53.

16. Li J, Alvarez B, Siwabessy J, Tran M, Huang Z, Przeslawski R, et al. Application of random forest, generalised linear model and their hybrid methods with geostatistical techniques to count data: Predicting sponge species richness. Environmental Modelling \& Software. 2017;97:112-29.

17. Li J, Tran M, Siwabessy J. Selecting Optimal Random Forest Predictive Models: A Case Study on Predicting the Spatial Distribution of Seabed Hardness. PLoS One. 2016;11(2):e0149089.

18. Bartz-Kurycki MA, Green C, Anderson KT, Alder AC, Bucher BT, Cina RA, et al. Enhanced neonatal surgical site infection prediction model utilizing statistically and clinically significant variables in combination with a machine learning algorithm. Am J Surg. 2018;216(4):764-77.

19. Breiman L. Random Forests. Machine Learning. 2001;45:5-32.

20. Touw WG, Bayjanov JR, Overmars L, Backus L, Boekhorst J, Wels M, et al. Data mining in the Life Sciences with Random Forest: a walk in the park or lost in the jungle? Brief Bioinform. 2013;14(3):315-26.

21. Liam A, Wiener M. Classification and regression by random forest. R News. 2002;2(3):18-22.

22. Ozcift A. Enhanced cancer recognition system based on random forests feature elimination algorithm. J Med Syst. 2012;36(4):2577-85.

23. Diaz-Uriarte R, Alvarez de Andres S. Gene selection and classification of microarray data using random forest. BMC Bioinformatics. 2006;7:3. 
24. Doerken S, Avalos M, Lagarde E, Schumacher M. Penalized logistic regression with low prevalence exposures beyond high dimensional settings. PLoS One. 2019;14(5):e0217057.

25. Sullivan LM, Massaro JM, D'Agostino RB, Sr. Presentation of multivariate data for clinical use: The Framingham Study risk score functions. Stat Med. 2004;23(10):1631-60.

26. Luo W, Phung D, Tran T, Gupta S, Rana S, Karmakar C, et al. Guidelines for Developing and Reporting Machine Learning Predictive Models in Biomedical Research: A Multidisciplinary View. J Med Internet Res. 2016;18(12):e323-e.

27. Streiner DL, Cairney J. What's under the ROC? An introduction to receiver operating characteristics curves. Can J Psychiatry. 2007;52(2):121-8.

\section{Figures}




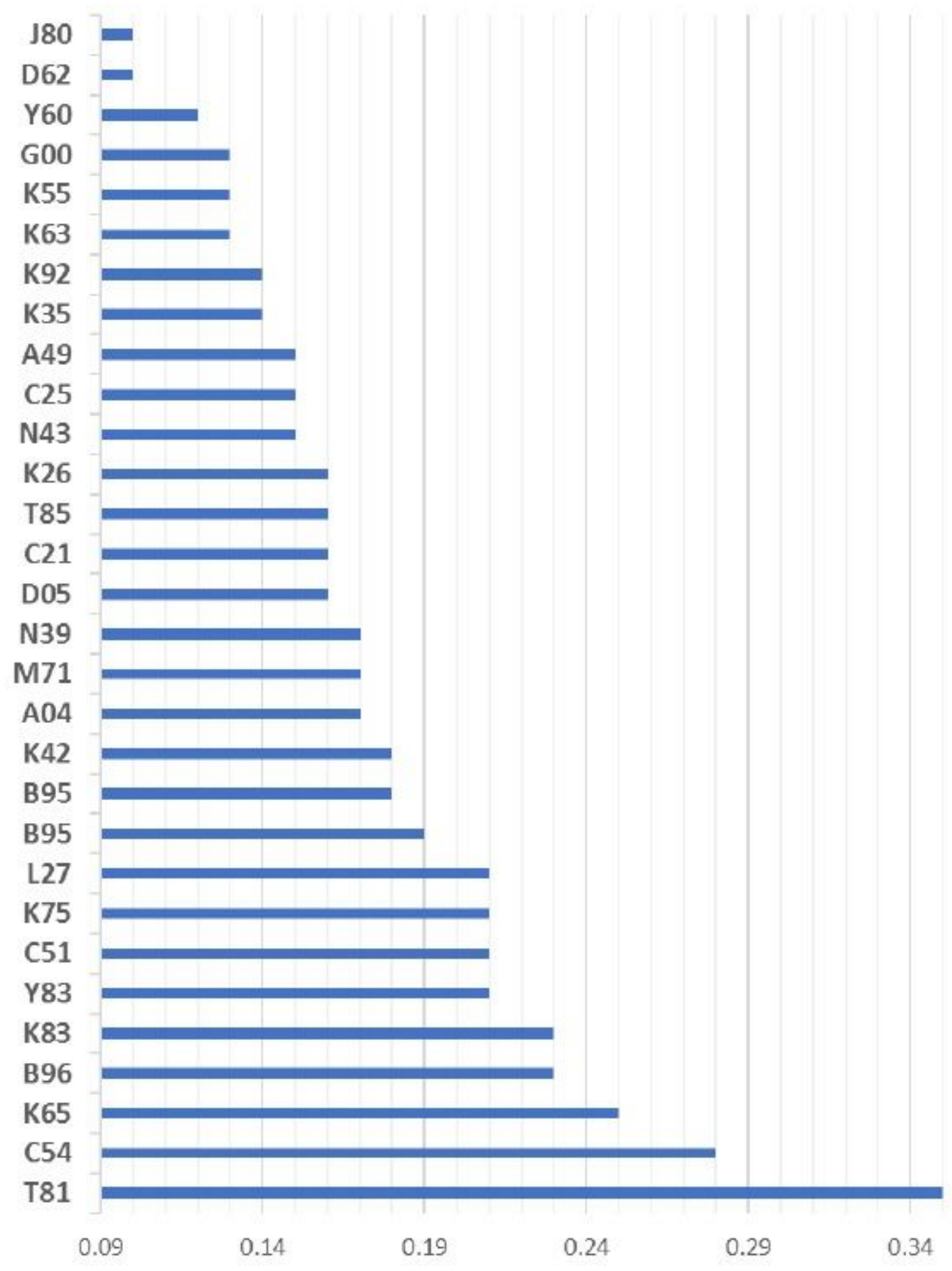

\section{Figure 1}

Description of tope 30 hospitalization diagnostic (ICD-10) codes to identify SSIs. T81 - Operative complication (infection, hemorrhage, etc.); C54 - Malignant neoplasm of specified part of uterus; K65 Peritonitis; B96 - Other bacterial agents as the cause of diseases classified elsewhere; K83 - Biliary duct infection, obstruction, perforation, or fistulation; Y83 - Surgical operation/procedures as the cause of abnormal reaction of the patient/or later complication; C51 - Malignant neoplasms of female genital 
organs; Y83 - Surgical operation/procedures as the cause of abnormal reaction of the patient/complication; C51 - Malignant neoplasms of female genital organs; K75 - Abscess of liver; L27 Dermatitis and eczema; B95 - Streptococcus and staphylococcus as the cause of diseases; K42 Umbilical hernia; A04 - Other bacterial intestinal infections; M71 - Bursal abscess, cyst, infection; N39 Other disorders of urinary system; D05 - Carcinoma in situ of breast; C21 - Malignant neoplasm of anus and anal canal; 785 - Complications of internal prosthetic devices, implants and grafts; K26 - Duodenal ulcer; N43 - Other disorders of prostate; C25 - Malignant neoplasm of pancreas; A49 - Bacterial infection of unspecified site; K35 - Acute appendicitis; K92 - Other diseases of digestive system; K63 - Other diseases of intestine; K55 - Vascular disorders of intestine; G00 - Bacterial meningitis, unspecified; Y60 Unintentional cut, puncture, perforation or haemorrhage during surgical and medical care; D62 - Acute posthaemorrhagic anemia; J80 - Acute respiratory distress syndrome 


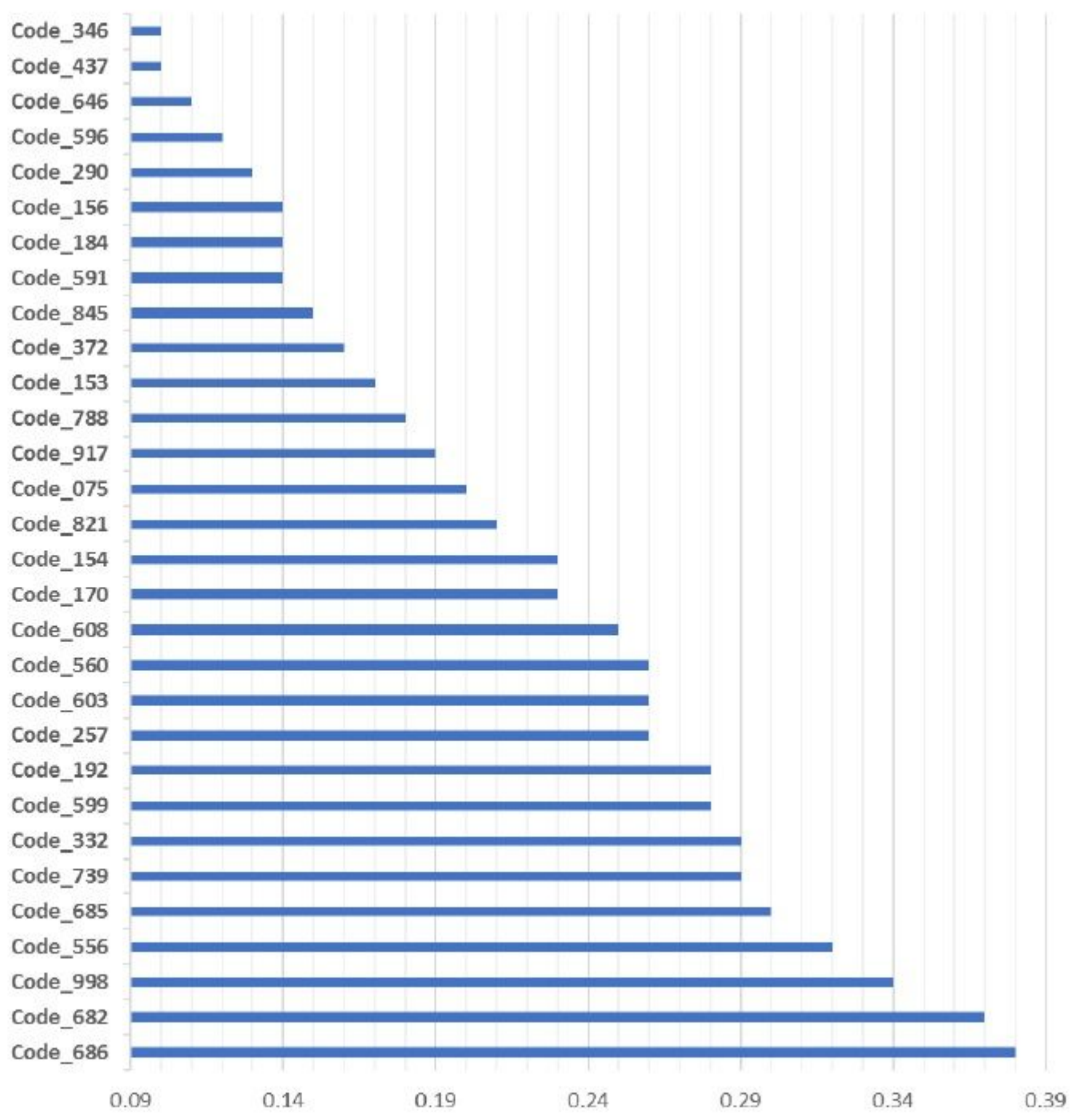

\section{Figure 2}

Description of the top 30 physician diagnostic (ICD-9) codes to identify SSIs. 686 - Pyoderma, pyogenic granuloma, other local skin infections; 682 - Cellulitis, abscess; 998 - Other complications of procedures, not elsewhere classified; 556 - Ulcerative colitis; 685 - Pilonidal cyst or abscess; 739 - Nonallopathic lesions, not elsewhere classified; 332 - Parkinson's disease; 599 - Other disorders of urethra and urinary tract; 192 - Malignant neoplasm of other and unspecified parts of nervous system; 257 - Testicular dysfunction; 603 - Hydrocele; 560 - Intestinal obstruction without mention of hernia; 608 - Other disorders 
of male genital organs; 170 - Malignant neoplasm of bone and articular cartilage; 154 - Malignant neoplasm of rectum, rectosigmoid junction and anus; 821 - Fracture of femur; 075- Infectious mononucleosis, glandular fever; 917- Superficial injury of foot and toe(s); 788 - Symptoms involving urinary system; 153 - Malignant neoplasm of large intestine - excluding rectum; 372 - Conjunctiva disorders (e.g., conjunctivitis, pterygium); 845 - Sprains and strains of ankle and foot; 591 Hydronephrosis; 184 - Malignant neoplasm of vagina, vulva, other female genital organs; 156 - Malignant neoplasm of gallbladder and extra hepatic bile ducts; 290 - Senile dementia, presenile dementia; 569Other disorders of intestine; 646 - Other complications of pregnancy (e.g., vulvitis, vaginitis, cervicitis, pyelitis, cystitis); 437- Other and ill-defined cerebrovascular disease; 346 - Other diseases of central nervous system (e.g., brain abscess, narcolepsy, motor neuron disease, syringomyelia)

Mean Decrease Accuracy

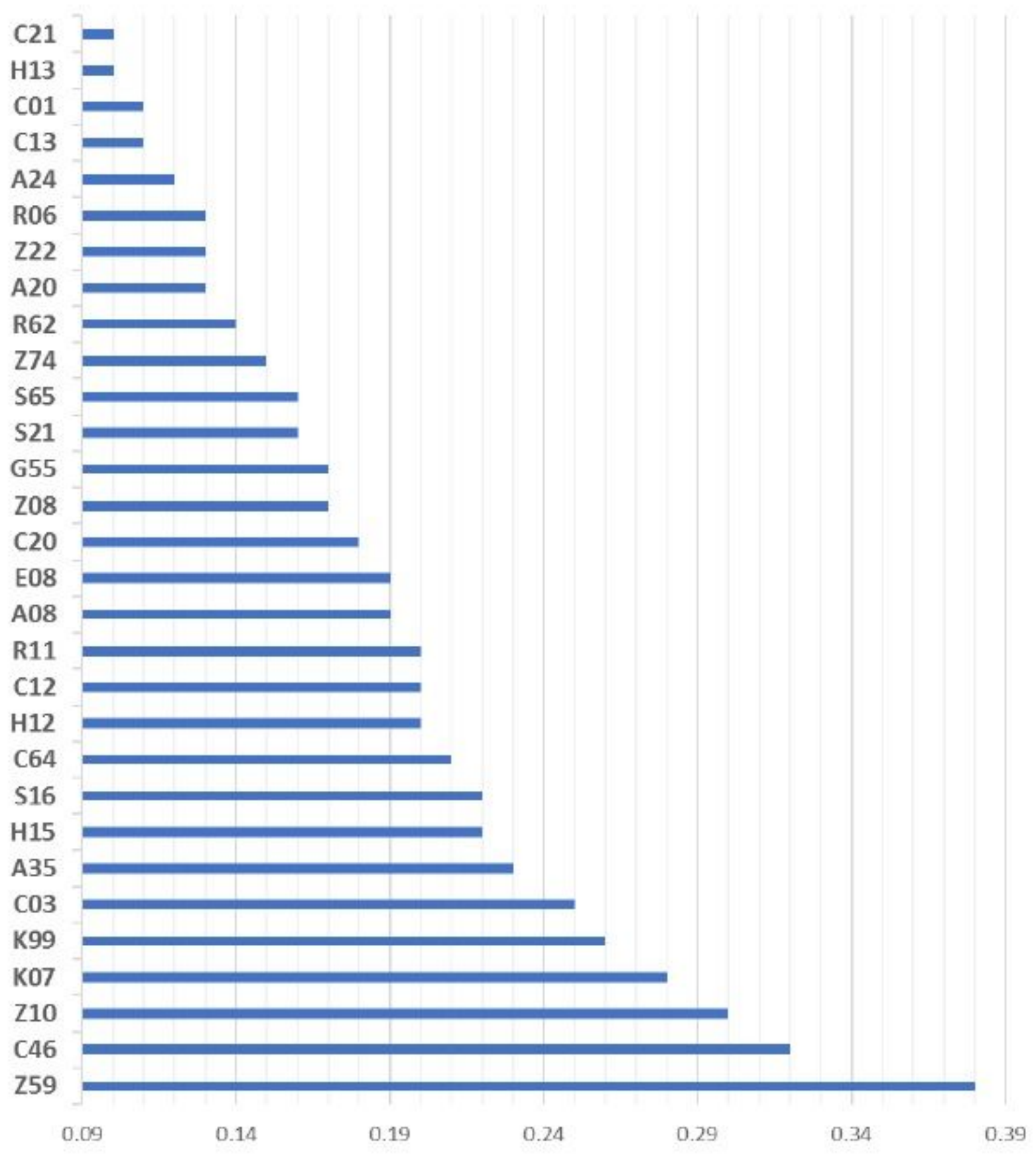




\section{Figure 3}

Description of the top 30 physician procedure claims to identify SSIs. Z59 - Digestive system surgical procedure; C46 - Infectious disease - non-emergency hospital in-patient services: assessment/ consultation; Z10 - Integumentary system surgical procedures: incision of abscess/ haematoma; K07 Family practice/geriatrics acute and chronic home care supervision; K99 - Emergency department special visit premium; C03 - General surgery, non- emergency hospital in-patient services-assessment, visits, consultations; A35 - Urology -consultations/ assessment; S16 - Digestive system surgical procedures; H15 - Family practice \& practice in general - weekend and holidays: assessment/care; C64 General thoracic surgery - non-emergency hospital in-patient services: consultation assessment; $\mathrm{H} 12$ Family practice \& practice in general - nights assessment and car; C12- Non-emergency hospital in-patient services: Subsequent visits by the MRP; R11- Integumentary system surgical procedures: operations of the breast; E08 - Hospital and institutional consultations/assessments by MRP; C20 - Obstetrics and gynecology - non-emergency hospital in-patient services; Z08 - Debridement of wound(s) and/or ulcer(s) extending into subcutaneous tissue, tendon, ligament, bursa and/or bone; G55- Diagnostic and therapeutic procedures, critical care; S21- Digestive system surgical procedures: rectum; S65 - Male genital surgical procedures; Z74 - Respiratory surgical procedures; R62- Musculoskeletal system surgical procedures - amputation; A20 - Obstetrics and gynecology - assessment or consultation; Z22 Musculoskeletal system surgical procedures; R06 - Myocutaneous, myogenous or fascia-cutaneous flaps, neurovascular island transfer, transplantation of free island skin and subcutaneous flap; A24 Otolaryngology - assessment/ consultation; C13 - Internal and occupational medicine: non- emergency hospital in-patient services; $\mathrm{CO} 1$ - Non-emergency hospital in-patient services, subsequent visits by the MRP; H13 - Family practice \& practice in general - weekdays, evenings: assessment/care; C21 Consultations/visits anaesthesia -non-emergency hospital in-patient services
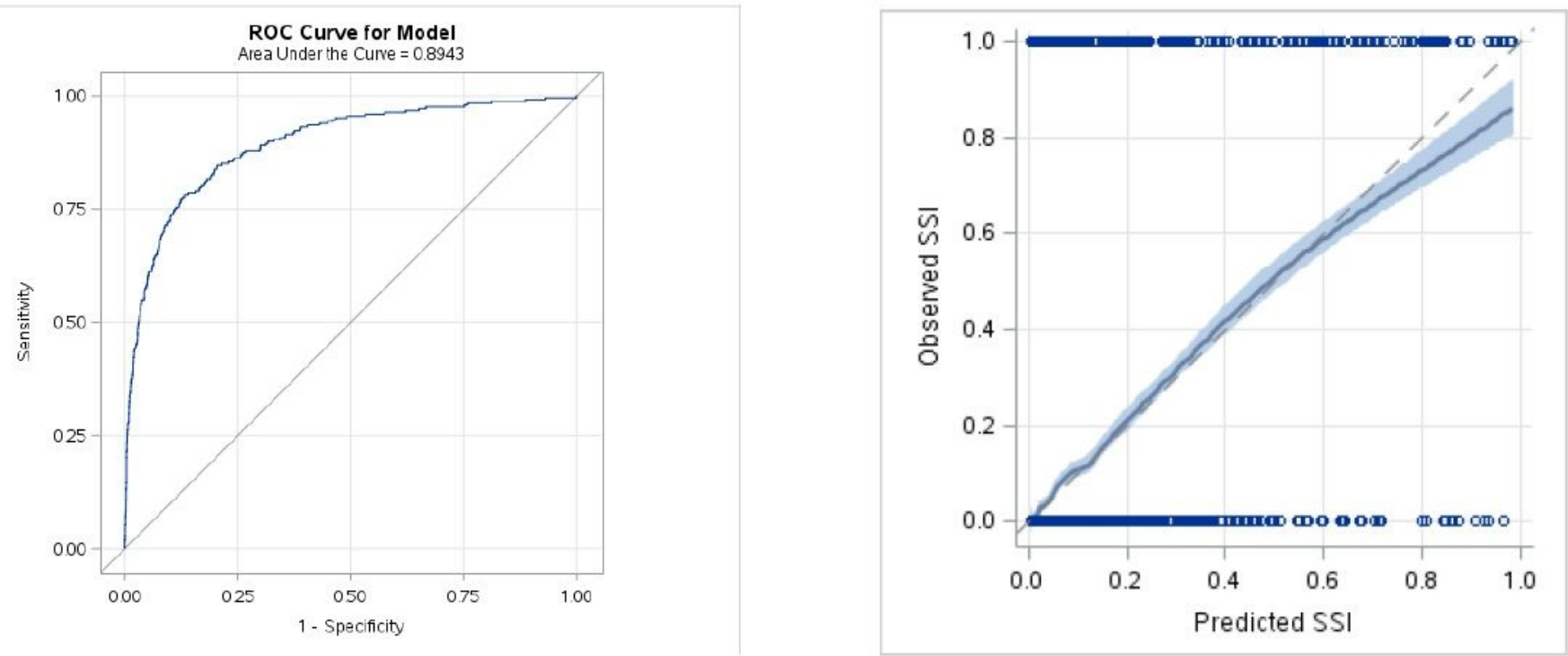

\section{Figure 4}


Receiver Operator Characteristics Curve (ROC curve) and *calibration plot for the full model with risk scores for hospitalization diagnostic (ICD-10) codes, physician diagnostic (ICD-9) codes, and physician procedure claims, adjusted for the **study covariates, in the validation cohort. *In the calibration plot, the observed percentage of patients having an SSI within 30 days of surgery is plotted against the predicted SSI risk from the SSI risk model (horizontal axis). **Study covariates: surgical specialties, age, sex, duration of surgery, emergency case, ASA class, concurrent surgical procedures

\section{Supplementary Files}

This is a list of supplementary files associated with this preprint. Click to download.

- Additionalfile1.DefinitionofdifferenttypesofSSIs.docx

- Additionalfile2.RandomForestsalgorithm.docx

- Additionalfile3.Datapartitioning.docx

- Additionalfile4.Descriptionofthestudycohort.docx

- Additionalfile5.Descriptionofderivationandvalidationsets.docx

- Additionalfile6.Titlepage.docx 